\title{
Fournier's Gangrene in a Neonate With Acute Myeloid Leukemia: A Case Report
}

\author{
Ziba Mosayebi, ${ }^{1,2}$ Ali Omidian, ${ }^{2}$ Amir Hossein Movahedian, ${ }^{1,3}$ Farzad Kompani, ${ }^{1,2}$ and Seyyed Saeed \\ Hosseininodeh ${ }^{2, *}$ \\ ${ }^{1}$ Department of Pediatrics, Tehran University of Medical Sciences, Tehran, IR Iran \\ ${ }^{2}$ Children's Medical Center, Pediatrics Center of Excellence, Tehran, IR Iran \\ ${ }^{3}$ Bahrami Children's Hospital, Tehran, IR Iran \\ "Corresponding author: Seyyed Saeed Hosseininodeh, Children's Medical Center, Pediatrics Center of Excellence, Tehran, IR Iran. E-mail: bonjour263@gmail.com
}

Received 2015 November 08; Revised 2015 December 21; Accepted 2016 January 23.

\begin{abstract}
Introduction: Fournier's gangrene is an infective necrotizing fasciitis of external genital and perineal region. Hematologic malignancies and immunocompromised status are predisposing factors. Simultaneous occurrence of Fournier's gangrene and congenital leukemia in neonates is extremely rare.

Case Presentation: We present a case of Fournier's gangrene in a 4-day-old female infant with a necrotic lesion in perineum and no history of trauma or other predisposing condition. Focusing on high blast percentage in blood cell count she was affected by acute myeloid leukemia (M4 type). Pseudomonas aeruginosa was isolated from the blood and wound culture. She was treated with broad spectrum antibiotics and supportive care. The parents refused chemotherapy and the patient was discharged from hospital. Bleeding and DIC was the cause of death in a local hospital few days later.

Conclusions: High index of suspicion is essential for diagnosis and appropriate treatment. Congenital leukemia should be considered in the differential diagnosis of a newborn with clinical features of sepsis and necrotizing fasciitis.
\end{abstract}

Keywords: Fournier's Gangrene, Acute Myeloid Leukemia, Neonate

\section{Introduction}

Fournier's gangrene, an infective necrotizing fasciitis of the perineal region, was first described by French venerologist Alfred Fournier $(1,2)$ in 1883 . This aggressive and life threatening disease is uncommon in children and very rare in neonates and has a mortality rate of near 50\% in this age group $(3,4)$. Prematurity, diaper rash, poor hygiene, trauma, anorectal diseases, urethral catheterization, circumcision, insect bite, strangulated hernia, omphalitis, systemic infections, immunocompromised status and hematologic malignancies are some predisposing factors in children $(1,4,5)$. Congenital leukemia defined as leukemia diagnosed in the first 28 days of life, is quite rare with an incidence of 4.7 cases per million births $(6,7)$. The prevalence of acute myeloid leukemia (AML) is more than acute lymphoblastic leukemia (ALL) in neonates, with acute myelomonocytic (M4) and monocytic (M5) being the most common subtypes. AML has better prognosis than ALL in neonates (8). The presentation of leukemia is typically with hepatosplenomgaly, leukemia cutis and/or hyperleukocytosis. Leukemia as an immune-suppressed condition is an extremely rare risk factor for necrotizing fasciitis in neonates. Here we describe a rare case of the si- multaneous occurrence of acute myeloid leukemia and Fournier's gangrene in a neonate.

\section{Case Presentation}

Our patient was a 4-day-old female with a birth weight of 3,100 grams and a gestational age of 39 weeks born from non-consanguineous parents. Her birth history was unremarkable. She was first admitted to a local hospital for hyperbilirubinemia started at the third day of life. There was no history of poor feeding and fever. She was not ill or toxic on primary examination. After one day of hospitalization, fever started and she was referred to our hospital for further evaluation. Clinical examination at admission revealed an axillary temperature of $39^{\circ} \mathrm{C}$, jaundice, hepatosplenomegaly and a necrotic lesion in perineum with swelling of the left labium major. Sharp margins of the lesion medially distended to the fourchette and anus and laterally to the origin of left thigh (Figure 1). There was no history of trauma or other predisposing condition. Initial diagnosis of necrotizing fasciitis and sepsis was made and she was treated by vancomycin and meropenem to cover both aerobic and anerobic organisms, in addition to other supportive measures. 


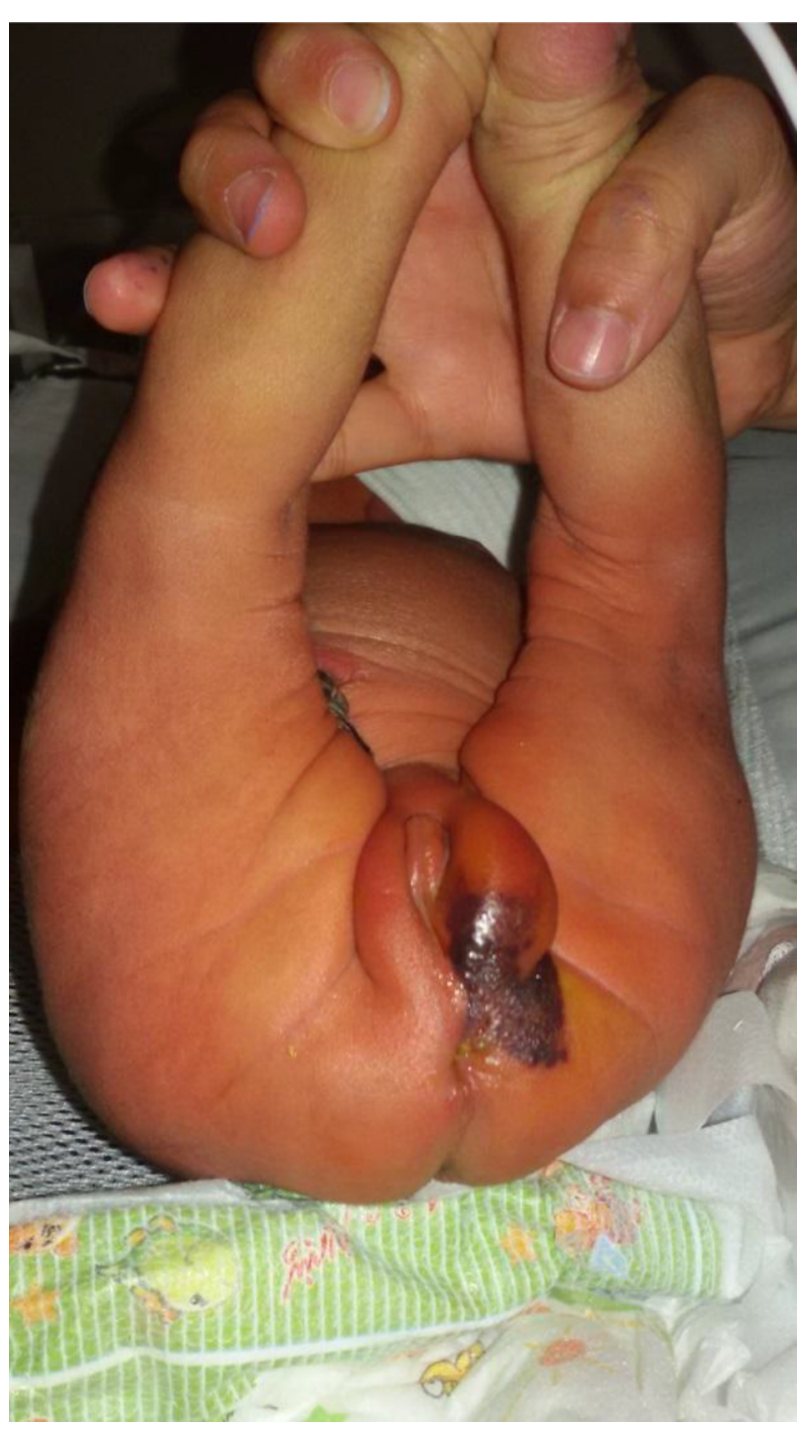

Figure 1. Skin Lesion in the First Examination

Laboratory study showed; direct hyperbilirubinemia (total bilirubin $17.6 \mathrm{mg} / \mathrm{dL}$, direct bilirubin $5.9 \mathrm{mg} / \mathrm{dL}$ ), blood cell count abnormality (WBC 2,160, neutrophil 5\% lymph 88\%, mono 7\%, Hb 12.8 gr/dL, Plt 21,000) and elevated CRP $41.9 \mathrm{mg} / \mathrm{L}$. TORCH study was negative. Blood and wound culture grew Pseudomonas aeruginosa sensitive to meropenem and amikacin.

In the course of treatment, by repeating $\mathrm{CBC}$ we found leukocytosis with a high percentage of blast cells and persistent thrombocytopenia (WBC 18,490, neut 5.7\%, lymph $15.1 \%$, mono $26 \%$, blast $52 \%$, myelocyte $1 \%$, RBC $2,800,000, \mathrm{Hb}$ 8.8 , Plt 29,000) despite proper antibiotic therapy. So a pediatrics hematologic consultation was requested to rule out malignancies. Bone marrow aspiration and flow cytometry was performed according to his suggestion. The result was; AML-M4 (Figure 2).

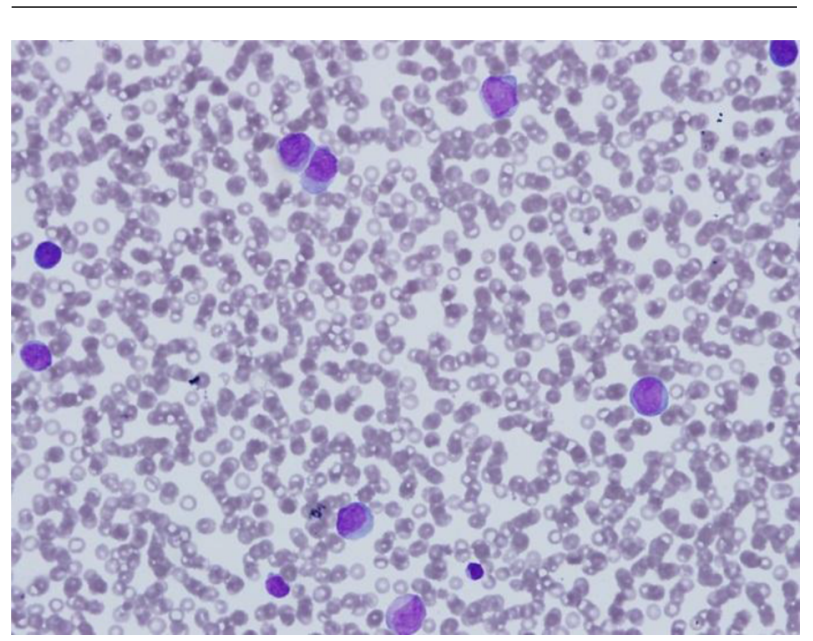

Figure 2. Blast Cells in Microscopic View of Bone Marrow

Skin lesion progressed from a superficial necrotic lesion to a deep soft tissue involvement in external genital area (Figure 3). Abnormal coagulation profile and poor patient's condition banned the surgeon from performing any surgical procedure for skin lesion, so local treatment with $\mathrm{N} / \mathrm{S}$ irrigation and sterile dressing continued. Parents refused chemotherapy and she was discharged against medical advice. Bleeding and DIC was the cause of her death in a local hospital few days later.

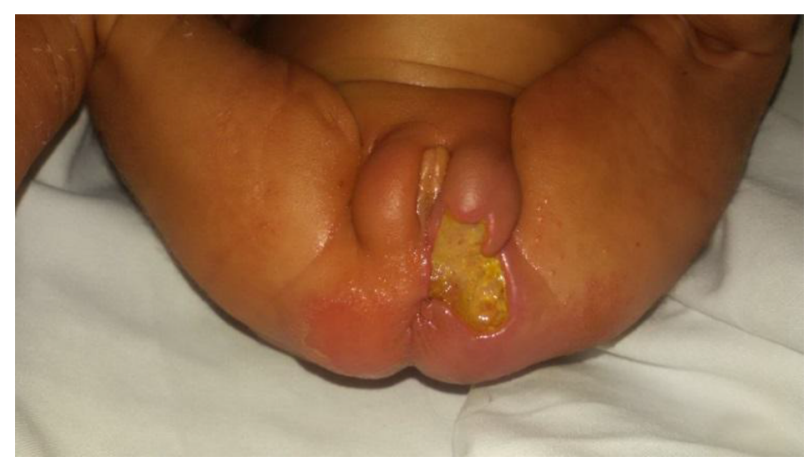

Figure 3. Skin Lesion After Antibiotic Therapy and Local Irrigation

\section{Discussion}

Fournier's gangrene (FG) is a fulminant form of infective necrotizing fasciitis that involves external genitals and 
perineal region. It is more common in adults but more than 56 pediatric cases have been reported so far, most of them being under 3 months of age $(1,2,9)$. Although the male to female ratio is reported to be 10: 1, our patient is a female. FG affects females as well as males and cases of vulvar necrosis have been reported (10). Predisposing factors vary with age. Diabetes mellitus, chronic alcoholism, malnutrition and immunosuppressive therapy are underlying disorders for affected adults whereas predisposing and etiological factors differ in neonates including prematurity, trauma, poor hygiene, systemic infections, insect bite, burns, circumcision, disorders of immune system, anorectal and periurethral diseases, strangulated hernia, phimosis, omphalitis, varicella infection, procedures in perineal region, instrumentation of urethra and hematologic malignancies $(1,2,5,11)$. Acute myeloid leukemia, M4, was the etiology of FG in our case. About 35 cases of FG associated with hematologic malignancies have been reported while about $88 \%$ of cases were complications of treatment and in few cases FG was the first sign of hematologic malignancies (12). Simultaneous occurrence of FG and congenital leukemia in neonates is very rare. Immune deficiency caused by leukemia could be the predisposing factor for FG. Lo reported an extensive necrotizing fasciitis in a neonate with AML (13). FG should be considered as the first sign of AML.

The cause of FG can be identified in approximately 95\% of cases $(1,11)$. There are two types of necrotizing fasciitis depending on the responsible organisms. Type 1 is usually polymicrobial including aerobic and anaerobic bacteria. Type 2 is monomicrobial and often caused by group A Streptococcus alone or in combination with Staphylococcus aureus (10). The usual organisms that cause Fournier gangrene in children are streptococci, staphylococci and anaerobes (1). In our patient, blood and wound culture yielded Pseudomonas aeruginosa. Cases of necrotizing fasciitis with Pseudomonas aeruginosa in pediatric patients affected by acute leukemia was reported by some researchers (13). Fustes-Morales et al. described $P$. aeruginosa as the most common organism isolated in $85 \%$ of necrotizing fasciitis cases. Association of $P$. aeruginosa and necrotizing fasciitis was also demonstrated by Al-Fifi et al. (14) in two cases and Rouzrokh et al. (15) in seven cases. Pseudomonas seems to be an important causative pathogen in neutropenic patients with a rapid and fatal course. Early diagnosis and prompt appropriate treatment is essential in FG. Treatment includes early IV-fluid therapy, hemodynamic stabilization, broad-spectrum antibiotics and surgical debridement of necrotic tissues. Although literature suggest aggressive debridement of wound but a recent study showed successful outcome by using more conservative and selective surgical methods (5). Hyperbaric oxy- gen is also used in some selected cases for the treatment of Fournier's gangrene (16). We started with a combination of broad spectrum antibiotics (meropenem and vancomycin) regimen and then modified it to meropenem and amikacin when culture results were available. Our patient was not a surgical candidate for debridement because of coagulopathy state and her poor condition, so we could only wash and keep the wound sterile. Mortality rate of Fournier's gangrene is ranged from 3 to 45 percent which is mostly due to severe sepsis, renal failure and coagulopathy (9). Despite all the medical and surgical advances in treatment the mortality remains high. We planned to start chemotherapy but the parents refused any type of treatment and the baby was discharged against medical advice.

Neonatal FG is extremely rare and there is a need for high index of suspicion to make the prompt diagnosis and early appropriate treatment. The physicians should consider Leukemia in the differential diagnosis of a newborn with clinical features of sepsis and necrotizing fasciitis.

\section{References}

1. Dey S, Bhutia KL, Baruah AK, Kharga B, Mohanta PK, Singh VK. Neonatal Fournier's gangrene. Arch Iran Med. 2010;13(4):360-2. [PubMed: 20597572].

2. Baghel B, Dhruv K. Fournier's gangrene in a neonate: A case report. J Nepal Paediatr Soc. 2010;30(2):166-7.

3. Zgraj O, Paran S, O'Sullivan M, Quinn F. Neonatal scrotal wall necrotizing fasciitis (Fournier gangrene): a case report. J Med Case Rep. 2011;5:576. doi: 10.1186/1752-1947-5-576. [PubMed: 22151925].

4. Ibekwe MU, Ojukwu JO, Ibekwe RC. Unusual presentation of necrotizing fasciitis in an HIV exposed infant: A case report. Nigerian JPaediatr. 2011;38(3):142-5.

5. Ekingen G, Isken T, Agir H, Oncel S, Gunlemez A. Fournier's gangrene in childhood: a report of 3 infant patients. J Pediatr Surg. 2008;43(12):e39-42. [PubMed: 19040919].

6. Ishii E, Oda M, Kinugawa N, Oda T, Takimoto T, Suzuki N, et al. Features and outcome of neonatal leukemia in Japan: experience of the Japan infant leukemia study group. Pediatr Blood Cancer. 2006;47(3):268-72. doi: 10.1002/pbc.20599. [PubMed:16333820].

7. Moura SV, Andrade F, Magalhaes IQ, Costa I, Silva DB, D’Andrea ML, et al. Clinical and molecular epidemiology of neonatal leukemia in Brazil. Leuk Lymphoma. 2015;56(4):903-9. doi: 10.3109/10428194.2014.938327. [PubMed: 24991719].

8. van der Linden MH, Creemers S, Pieters R. Diagnosis and management of neonatal leukaemia. Semin Fetal Neonatal Med. 2012;17(4):1925. [PubMed: 22510298].

9. Eke N. Fournier's gangrene: a review of 1726 cases. $\mathrm{Br} J$ Surg. 2000;87(6):718-28. doi: 10.1046/j.1365-2168.2000.01497.x. [PubMed: 10848848].

10. Mehl AA, Nogueira Filho DC, Mantovani LM, Grippa MM, Berger R, Krauss D, et al. Management of Fournier's gangrene: experience of a university hospital of Curitiba. Rev Col Bras Cir. 2010;37(6):435-41. [PubMed: 21340259].

11. Mukoro Duke G, Tabowei BI, Olatoregun F. Neonatal Fournier's gangrene; sequelly of traditional birth practice: Case report and short review. J Dental Med Sci. 2013;5(3):01-3.

12. D'Arena G, Pietrantuono G, Buccino E, Pacifico G, Musto P. Fournier's gangrene complicating hematologic malignancies: A case report 
and review of licterature. Mediterr J Hematol Infect Dis. 2013;5(1) doi: 10.4084/MJHID.2013.067. [PubMed: 24363882].

13. Negosanti L, Aceti A, Bianchi T, Corvaglia L, Negosanti F, Sgarzani $\mathrm{R}$, et al. Adapting a vacuum assisted closure dressing to challenging wounds: Negative pressure treatment for perineal necrotizing fasciitis with rectal prolapse in a newborn affected by acute myeloid leukaemia. Eur J Dermatol. 2010;20(4):501-3. doi: 10.1684/ejd.2010.0964. [PubMed: 20406723].
14. Al-Fifi S, Al Azraqi T, Al Hasan I. Fatal necrotizing fasciitis caused by pseudomonas aeruginosa in infants. Bahrain Med Bull. 2010;32(3):1-4

15. Rouzrokh M, Tavassoli A, Mirshemirani A. Fournier's gangrene in children: Report on 7 cases and review of literature. Iran J Pediatr. 2014;24(5):660-1. [PubMed: 25793080].

16. Safioleas MC, Stamatakos MC, Diab AI, Safioleas PM. The use of oxygen in Fournier's gangrene. Saudi Med J. 2006;27(11):1748-50. [PubMed: 17106556]. 